\title{
Evaluation of Mid-Plane Dose by Flat Panel Transit Dosimetry Method and by EBT2 Film Incident Dosimetry
}

\author{
Kalliopi Mandourari ${ }^{1}$, Christos Antypas' ${ }^{1}$, Christina Armpilia' ${ }^{1}$, Ioannis Floros ${ }^{1}$, \\ Pantelis Karaiskos ${ }^{2}$, Panagiotis Sandilos ${ }^{1}$ \\ ${ }^{1}$ Radiology Department, Aretaieion Hospital, National \& Kapodistrian University of Athens, Athens, Greece \\ ${ }^{2}$ Medical Physics Laboratory, Medical School, University of Athens, Athens, Greece \\ Email: p.mandourari@yahoo.com
}

Received 8 July 2015; accepted 8 August 2015; published 11 August 2015

Copyright (C) 2015 by authors and Scientific Research Publishing Inc.

This work is licensed under the Creative Commons Attribution International License (CC BY).

http://creativecommons.org/licenses/by/4.0/

c) (i) Open Access

\begin{abstract}
Purpose: The aim of this study is to validate an easily applicable flat panel dosimetry method based on the back projection approach and to compare this method with the incident dosimetry by EBT2 film method for mid-plane dose calculations. Methods: The dosimetric characteristics of the flat panel were determined for $6 \mathrm{MV}$ photon energy. Then, the methodology to calculate the dose on the central axis of the photon beam was described. While, the flat panel dosimetry method was validated with phantom measurements using an ionization chamber. Once the method was validated, in vivo measurements of ten prostate patients treated with $6 \mathrm{MV}$ photon energy 3D conformal plans were also performed both with the flat panel and the EBT2 films. Results: The phantom measurements revealed a mean dispersion of $1.7 \%$ between flat panel and ionization chamber doses and $2.2 \%$ between flat panel and EBT2 film doses. While, the in vivo measurements in prostate patients revealed a mean dispersion of $0.8 \%$ between flat panel doses and treatment planning calculated doses and 1\% between flat panel and EBT2 film doses. Conclusions: The presented flat panel dosimetry method is accurate, easily applicable to all types of flat panels without the use of any sophisticated software and is not time consuming.
\end{abstract}

\section{Keywords}

EPID, EBT2 Films, In Vivo Dosimetry, Calibration Model

\section{Introduction}

The major challenge that must be contented in the treatment of cancer is the irradiation of the tumor with a high

How to cite this paper: Mandourari, K., Antypas, C., Armpilia, C., Floros, I., Karaiskos, P. and Sandilos, P. (2015) Evaluation of Mid-Plane Dose by Flat Panel Transit Dosimetry Method and by EBT2 Film Incident Dosimetry. International Journal of Medical Physics, Clinical Engineering and Radiation Oncology, 4, 243-254. http://dx.doi.org/10.4236/ijmpcero.2015.43029 
dose, while the surrounding healthy tissues suffer as little radiation damage as possible. Because of the increasing complexity of all steps in contemporary radiotherapy, the requirement for a thorough verification of the dose attributed to the patient is higher [1]. In vivo dose verification is used to reveal and prevent major deviations between the prescribed dose and the dose actually delivered to the patient. This method is usually performed by placing the classic dosimeters (diodes, TLDs, MOSFETs, radiochromic films) on the skin or inside the patient to derive the dose at specific points inside the patient [2]. However, in recent years the Electronic Portal Imaging Devices (EPIDs) are also used for dosimetric verification purposes and are considered superior to "traditional" dosimeters [3]-[5].

Dose verification with EPID is associated with accurate evaluation of the EPID response to different treatment conditions and parameters. The implementation of a dosimetric calibration model is necessary to convert a grayscale EPID image into a transit dose image. This calibration model may be used either with a back-projection algorithm or with the "forward approach" [1]. However, according to AAPM Task Group 58 the determination of this calibration model depends on various treatment parameters affecting image quality (beam energy, number of MUs, collimator settings, patient thickness, detector distance) [6]. For this purpose, flat panel inherent and dosimetric characteristics should be firstly investigated and determined. Once the calibration model and the algorithm are defined, the accuracy of the method should be evaluated in a phantom before used clinically.

The study presented is the validation of an easily applicable Siemens EPID verification method based on the back-projection approach and the comparison of this method with the EBT2 film in vivo verification method. The method was tested with phantom measurements using an ionization chamber and compared with the EBT2 film method. Once the method was validated it was applied on ten prostate patients treated with 3D conformal plans. Furthermore, EBT2 films were implemented for comparison.

\section{Methods and Materials}

In this study, an a-Si flat panel EPID (OptiVue 500 Siemens Medical Solutions, Concord, CA) attached to an Oncor Impression linear accelerator (Siemens Medical Solutions, Concord, CA) was investigated. The LINAC (Linear Accelerator) produces a $6 \mathrm{MV}$ photon beam and is equipped with a multileaf collimator (Optifocus, Siemens, Germany) of 82 leaves and a virtual wedge option. The flat panel has a $41 \times 41 \mathrm{~cm}^{2}$ active detection area $(512 \times 512$ pixels) yielding a pixel resolution of $0.8 \mathrm{~mm}$. The Source-Imager Distance (SID) can vary between 115 and $160 \mathrm{~cm}$, while lateral and longitudinal movements of the panel are not possible.

All measurements in this study were performed in the clinical dose rate mode of $250 \mathrm{MU} / \mathrm{min}$, while the field size dimensions are defined at $\mathrm{SAD}=100 \mathrm{~cm}$ and the SID was set at $150 \mathrm{~cm}$. In clinical mode, the use of EPID and acquisition of imaging data were made with the Siemens Coherence Therapist Workspace version 2.0.125. All EPID measurements were made in the "Port During" acquisition mode, which means that imaging data acquisition starts with beam-on and stops when the beam turns off. An EPID image is stored as a two-dimensional (2D) accumulated pixel value distribution averaged over all sub frames. The integration time per sub frame is $285 \mathrm{~ms}$ resulting in a constant frame acquisition rate of 3.5 frames per second.

The reference central dose measurements were performed with an ionization chamber (Farmer FC65-G 0.6 $\mathrm{cm}^{3}$ ) connected to a Dose 1 (Wellhofer-Scanditronix) electrometer. While, the reference patient doses were given by the Oncentra treatment planning system. Finally, the film verification measurements were performed with Gafchromic ${ }^{\circledR}$ EBT2 films which were scanned with an Epson Perfection V750 Pro.

Analysis of the EPID and EBT2 images is accomplished using the programming language MATLAB 7.1.

\subsection{Flat Panel Inherent Characteristics}

\subsubsection{EPID Signal and Response}

The EPID signal $\left(S_{\text {EPID }}\right)$ is defined as the average pixel value anywhere on the 2D EPID image multiplied by the number of frames acquired [4].

$$
S_{\mathrm{EPID}}=\text { Average pixel value } \times \# \text { frames }
$$

The EPID response $\left(R_{\mathrm{EPID}}\right)$ is defined as the ratio of the EPID signal, $S_{\mathrm{EPID}}$, in a central region of interest (ROI) of $10 \times 10 \mathrm{~mm}^{2}$ to the dose delivered to the device at that area [4]:

$$
R_{\mathrm{EPID}}=\frac{S_{\mathrm{EPID}}}{D_{\text {ref }}}
$$




\subsubsection{Buildup}

The implementation of adequate buildup layer is important in EPID dosimetry not only for the establishment of the required electron equilibrium but also for the attenuation of the low energy photons scattered from the patient especially at small patient-detector air gaps [3] [4] [7] [8].

The effective layer of the OptiVue 500 does not provide adequate electronic equilibrium. The additional buildup layer of solid water material was determined with successive EPID signal measurements with respectively increasing thickness of $0 \mathrm{~mm}$ to $1.5 \mathrm{~cm}$ solid water. The EPID signal was measured for a field size of $10 \mathrm{~cm} \times 10 \mathrm{~cm}$ and 100 MUs per irradiation in free air. The additional buildup layer which corresponds to the maximum EPID signal was determined. The maximum EPID signal measurement corresponds to the additional buildup layer was found. This additional buildup layer was placed on top of the flat panel in all subsequent measurements.

\subsubsection{Image Lag and Ghosting}

Amorphous silicon EPIDs suffer from image lag and gain ghosting effects according to several authors [3] [4] [9]-[11]. In order to determine the ghosting effects on the Optivue 500, the flat panel and the ionization chamber were irradiated under the same conditions with 5 - 300 MUs using a field size of $10 \mathrm{~cm} \times 10 \mathrm{~cm}$ in free air [3] [4] [9] [11]. The ghosting correction factor $G(t)$ as a function of beam time is then determined according to McDermott et al. [4].

\subsubsection{EPID Dose Calibration and Field Size Dependence}

Amorphous silicon EPIDs suffer from ghosting effects resulting in non-linear EPID response [3] [4] [9]-[11]. The EPID signal ( $\left.S_{\text {EPID }}\right)$ corrected for the ghosting effects is then given by the following formula:

$$
S_{\text {EPID_G }}=\frac{\text { Average pixel value } \times \# \text { frames }}{G(t)}
$$

where $G(t)$ is the ghosting correction factor as a function of irradiation time $t$.

EPID dose calibration was performed with the flat panel and the ionization chamber irradiated under the same conditions [4] [8] [9] [11]-[14]. Dose measurements for a field of $10 \times 10 \mathrm{~cm}^{2}$ in air with successive irradiations of 5, 10, 20,60, 80, 100, 200 and 300 MUs were performed. The EPID dose in air for a $10 \times 10$ $\mathrm{cm}^{2}$ irradiation field is then given by the following formula:

$$
D_{\text {EPID }}^{\text {AIR }}(10)=S_{\text {EPID_G }} \times C_{F}
$$

where $S_{\text {EPID_G }}$ is the corrected EPID signal in pv (pixel value) and $C_{F}$ is the EPID calibration factor in cGy/pv.

In order to determine the field size dependence the flat panel and the ionization chamber were irradiated under the same conditions in free air for field sizes of $5 \times 5 \mathrm{~cm}^{2}-20 \times 20 \mathrm{~cm}^{2}$ and a total amount of $50 \mathrm{MUs}$ for each field size.

The dose measured by flat panel in air for a given field size $\mathrm{w}$ is given by the following relationship:

$$
D_{\text {EPID_cor }}^{\text {AIR }}(w)=D_{\text {EPID_uncor }}^{\text {AIR }}(w) \div S_{\text {EPID }}^{\text {AIR }}(w)
$$

where $D_{\mathrm{EPID} \text { uncor }}^{\mathrm{AIR}}(w)$ is the dose measured by the EPID in air without the correction for the scattered radiation and $S_{\mathrm{EPID}}^{\mathrm{AR}}(w)$ is defined as the EPID air scatter factor and takes into account the scattered radiation for the different field sizes.

\subsection{Back Projection Formalism for EPID Dosimetry}

The presence of an attenuator in the beam path results in absorption of the radiation reaching the EPID. In this situation, EPID records the transit dose and the effect of the attenuator can be expressed by an attenuation factor depending on the attenuator thickness and the field size $(w)$.

The attenuation factor $A F(w, t)$ defined as the ratio of the transit dose $D_{\text {EPID_uncor }}^{\text {TRANIT }}(w)$ to the dose measured in free air $D_{\mathrm{EPID} \_ \text {uncor }}^{\text {AIR }}(w)$ is given by:

$$
A F(w, t)=\frac{D_{\text {EPID_uncor }}^{\text {TRANSIT }}(w)}{D_{\text {EPID_uncor }}^{\text {AR }}(w)}
$$


Thus, the transit dose is expressed by the following formalism:

$$
D_{\text {EPII_uncor }}^{\mathrm{TRANIT}}(w)=D_{\mathrm{EPII} \_ \text {uncor }}^{\mathrm{AIR}}(w) \times A F(w, t)
$$

To determine the attenuation factor $A F(w, t)$, measurements were performed with the flat panel in free air and with attenuator of various thicknesses $(t=10 \mathrm{~cm}, 15 \mathrm{~cm}, 20 \mathrm{~cm}, 25 \mathrm{~cm})$, field sizes from $5 \times 5 \mathrm{~cm}^{2}$ to $20 \times 20$ $\mathrm{cm}^{2}$ at $S S D=100 \mathrm{~cm}$ and a total amount of 50 MUs for each field size-thickness combination.

In clinical applications, the transit dose is recorded by the EPID. Using formula (7) the $D_{\mathrm{EPID} \_ \text {uncor }}^{\mathrm{AIR}}(w)$ is calculated and corrected for the scattered radiation $S_{\mathrm{EPID}}^{\mathrm{AIR}}(w)$. The $D_{\mathrm{EPID} \_c o r}^{\mathrm{AIR}}(w)$ is then transformed to the surface dose on the attenuator using the inverse square law.

Finally, the dose at depth $\mathrm{d}$ inside the attenuator can be calculated as follows:

$$
D_{P}^{\mathrm{EPID}}(d)=D_{\mathrm{EPII} \_ \text {cor }}^{\mathrm{AIR}}(w) \times\left(\frac{S I D}{S S D}\right)^{2} \times P D D(d, w, S S D)
$$

or

$$
D_{P}^{\mathrm{EPID}}(d)=\frac{D_{\mathrm{EPID} \text { uncor }}^{\mathrm{TRANIT}}(w)}{S_{\mathrm{EPID}}^{\mathrm{AIR}}(w) \times A F(w, t)} \times\left(\frac{S I D}{S S D}\right)^{2} \times P D D(d, w, S S D)
$$

where $D_{P}^{\mathrm{EPID}}(d)$ is the dose at the point of interest as reconstructed by the EPID measurement, $D_{\mathrm{EPID} \text { cor }}^{\mathrm{AIR}}(w)$ is the dose measured in air for a $w$ field size by the EPID corrected for the scattered radiation and $\operatorname{PDD}(\bar{d}, w, S S D)$ is the corresponding percent depth dose.

\subsection{Formalism for EBT2 Film Dosimetry}

EBT2 film handling was performed according to the recommendations by Alva $\mathrm{H}$ et al. [15]. EBT2 films were cut in $5 \times 5 \mathrm{~cm}^{2}$ pieces for dose calibration and patient in vivo measurements. All films, prior to irradiation were scanned (RGB format, 150 dpi) with an EPSON perfection V750pro, for pre-irradiation background measurement [16]. EBT2 dose calibration was performed by placing individual precut films in a homogeneous solid water phantom at $10 \mathrm{~cm}$ depth on the central axis of the beam. All films were irradiated with a $10 \times 10 \mathrm{~cm}^{2}$ field and 50 - 350 MUs while the corresponding reference dose $D_{\text {ref }}$ was measured with a Farmer FC65G ionization chamber positioned at the same irradiation conditions with that of EBT2 films. Post-irradiation film scanning was performed 24 hours after irradiation [17] while the TIFF images obtained were further analyzed with the program MATLAB 7.1 on the three color channels (RGB).

According to the IAEA recommendations, an appropriate buildup layer should be used in radiochromic film dosimetry for electronic equilibrium [2]. However, in this study a buildup layer was not used, because measurements were performed simultaneously with the EBT2 film placed on the surface of the phantom (patient) and the flat panel. The existence of the buildup layer would affect the measurement of flat panel due to absorption.

The lack of electronic equilibrium, because of the buildup layer absence, was balanced with the introduction of the conversion factor of surface to maximum dose.

The conversion factor of surface to maximum dose $\left(C F_{\text {Surface Dose } \rightarrow D_{\max }}\right)$ was determined:

$$
C F_{\text {Surface Dose } \rightarrow D_{\max }}=\frac{D_{\max }}{D_{\text {Surface }}}
$$

It is defined as the ratio of the maximum dose to the surface dose on the phantom or patient for a given field size $w$ measured by the EBT2 films. To determine $C F_{\text {Surface Dose } \rightarrow D_{\max }}$ EBT2 films were placed simultaneously on the surface and at the depth of maximum dose of a homogenous solid water phantom on the central axis of the beam and were irradiated with fields ranging from $5 \times 5 \mathrm{~cm}^{2}$ to $20 \times 20 \mathrm{~cm}^{2}$ at $S S D=100 \mathrm{~cm}$ and a total amount of 200 MUs for each irradiation. $C F_{\text {Surface Dose } \rightarrow D_{\max }}$ was measured in homogenous solid water phantom of thicknesses $10 \mathrm{~cm}$ and $20 \mathrm{~cm}$.

Finally, the dose at the point of interest $(d)$ inside the phantom or patient is given by the following expression:

$$
D_{P}^{\mathrm{EBT2}}(d)=D_{\text {Surface }} \times C F_{\text {Surface Dose } \rightarrow D_{\max }} \times P D D(d, w, S S D)
$$


where $D_{P}^{\mathrm{EBT2}}(d)$ is the dose at the point of interest as reconstructed by the EBT2 film measurement, $D_{\text {Surface }}$ is the EBT2 film measured dose on the surface of the phantom or patient, $C F_{\text {Surface Dose } \rightarrow D_{\max }}$ is the conversion factor of surface dose to maximum dose and $\operatorname{PDD}(d, w, S S D)$ is the corresponding percent depth dose.

\subsection{The Mid-Plane Dose by Phantom Verification Measurements}

The proposed EPID dosimetry method was verified with measurements in a homogeneous solid water phantom. For this purpose, a homogeneous solid water phantom of two different thicknesses $(10 \mathrm{~cm}, 20 \mathrm{~cm})$ was scanned by CT and transferred by DICOM to TPS. The mid-plane dose was calculated by TPS for field sizes of $5 \times 5$ $\mathrm{cm}^{2}-20 \times 20 \mathrm{~cm}^{2}$ and 200 MUs at $S S D=100 \mathrm{~cm}$. The above irradiation conditions were simulated and delivered with linac beam and the transit dose was recorded by the EPID. Mid-plane dose $D_{P}^{\mathrm{EPID}}(d)$ as reconstructed by the EPID transit dose was calculated by applying the formula (8). Moreover, surface dose $\left(D_{\text {surface }}\right)$ was measured with EBT2 films on the phantom surface and mid-plane dose $D_{P}^{\mathrm{EBT2}}(d)$ as reconstructed by the EBT2 surface measured dose was then calculated by applying the formula (10). Mid-plane dose was also measured with an ionization chamber placed at point of interest inside the phantom. Finally, all the above mid-plane doses, either reconstructed or measured, were compared to the mid-plane doses as calculated by the TPS.

\subsection{In Vivo Measurements}

Finally, in vivo dosimetry measurements of ten 3D conformal prostate treatment plans were implemented. Prostate was treated using the four field isocentric box technique with the patient lying in supine position. Measurements were performed for the gantry $0^{\circ}$ field for three fractions (total 30 beams). Measurements were performed only for the gantry $0^{\circ}$ field to avoid affecting the department's workflow. Each field was represented by its equivalent square field. Patient's thickness was measured on the CT image (AP distance). Simultaneously, the transit dose $D_{\mathrm{EPID} \_ \text {uncor }}^{\mathrm{TRANST}}(w)$ and the surface dose $\left(D_{\text {Surface }}\right)$ were measured by the EPID and the EBT2 film respectively. Subsequently, reconstructed doses at the isocenter were calculated by applying the formulas (8) and (10). Each reconstructed isocenter dose, either by the EPID or the EBT2 films, was compared to the corresponding calculated dose by TPS. In this study the IAEA tolerance level of 5\% was considered appropriate [2]. When the in vivo results were out of the $\pm 5 \%$ the physicists investigated the patient setup, the machine settings and the TPS calculations in order to determine possible errors.

\section{Results}

\subsection{Flat Panel Inherent Characteristics}

\subsubsection{Buildup}

The maximum EPID response was measured for an additional buildup layer of $0.4 \mathrm{~cm}$ thickness of solid water (RW3). Hence, all the subsequent measurements were performed under buildup conditions with this additional buildup layer placed on the surface of EPID.

\subsubsection{Image Lag and Ghosting}

Figure 1 shows the normalized EPID response (points set) as a function of irradiation time without taking into account any correction and the ideal response (dashed line). It is obvious that in short irradiation times the measured response remains significantly below the ideal. Specifically, in irradiation times between 20 and 300 MUs the linearity is within 3\%, while below 20 MUs linearity shows significant divergence due to the ghosting effects (Figure 1). In order to correct the EPID response for this divergence a ghosting correction factor $G(t)$ is used. According to McDermott et al. [4] the ghosting correction factor can be exponentially fitted by the normalized $R_{\mathrm{EPID}}$ as a function of irradiation time (Figure 1 ). The ghosting factor is then determined as follows:

$$
G(t)=a_{0}-a_{1} \exp \left(-b_{1} t\right)-a_{2} \exp \left(-b_{2} t\right)-a_{3} \exp \left(-b_{3} t\right)-a_{4} \exp \left(-b_{4} t\right)
$$

where $a_{0}=1, a_{1}=0.04646, a_{2}=-0.006748, a_{3}=-0.09769, a_{4}=0.4355$ are amplitudes and $b_{1}=0.04849 \mathrm{~s}^{-1}, b_{2}=0.8777 \mathrm{~s}^{-1}, b_{3}=0.3362 \mathrm{~s}^{-1}, b_{4}=0.6823 \mathrm{~s}^{-1}$ are decay rates according to our fitted data.

After applying the ghosting correction factor the linearity is within $\pm 2 \%$ from 5 to 300 MUs.

The ghosting correction factor $G(t)$ was applied in all subsequent measurements. 


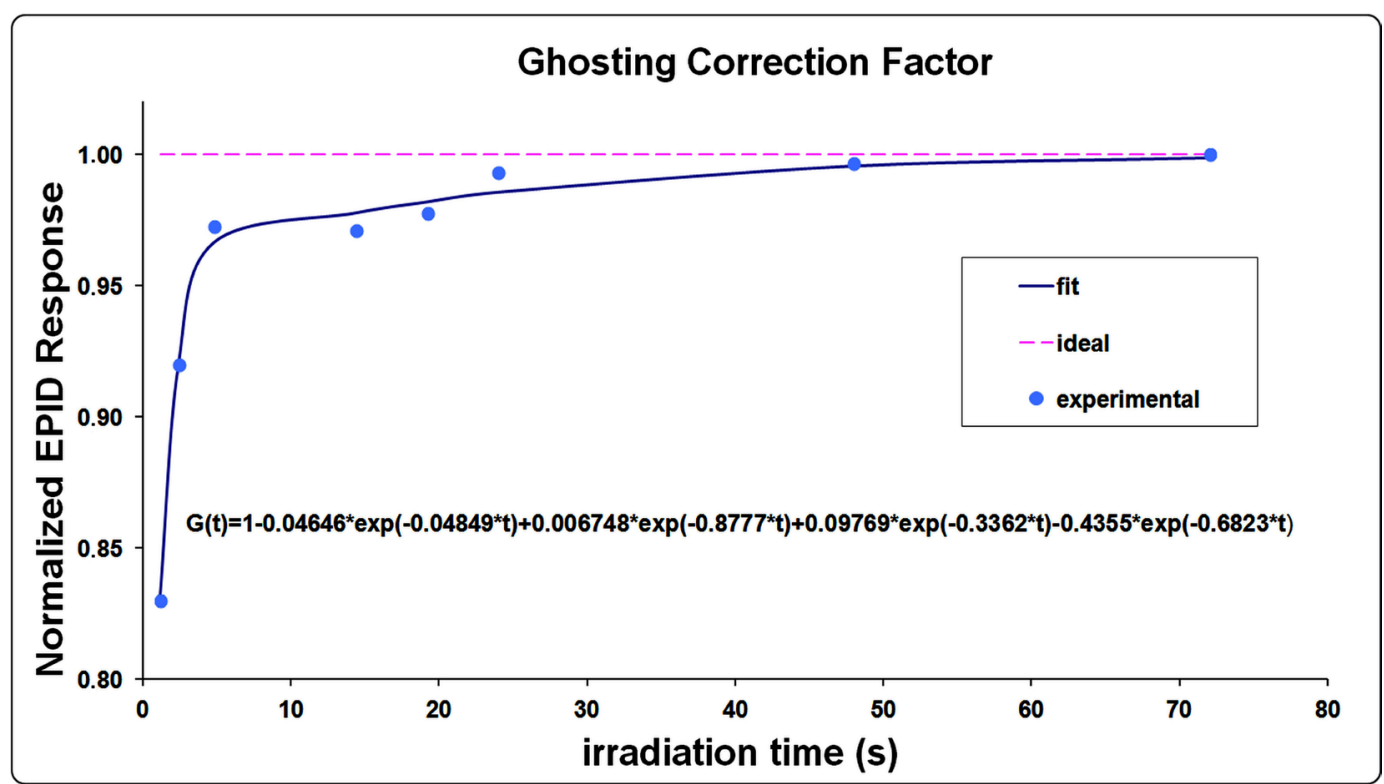

Figure 1. Determination of ghosting correction factor. The normalized EPID response as a function of irradiation time and the ideal response (dashed line).

\subsubsection{EPID Dose Calibration and Field Size Dependence}

According to our measurements the EPID calibration factor $\left(C_{F}\right)$ is $C_{F}=2.92124 \times 10^{-5} \mathrm{cGy} / \mathrm{pv}$.

While in Figure 2 the dose measured in air by the a-Si detector $D_{\text {EPID_uncor }}^{\text {AIR }}(w)$ and the ionization chamber on the central axis as a function of field side is presented. All data are normalized to the dose delivered by the $10 \times 10 \mathrm{~cm}^{2}$ field. According to our measurements, the EPID response differs significantly from the ionization chamber response. Deviations up to $6.7 \%$ were observed for $20 \times 20 \mathrm{~cm}^{2}$ field size. In order to correct the EPID response for field sizes different from $10 \times 10 \mathrm{~cm}^{2}$ the EPID air scatter factor $S_{\mathrm{EPID}}^{\mathrm{AIR}}(w)$ is used. $S_{\mathrm{EPID}}^{\mathrm{AIR}}(w)$ is the ratio of the dose measured by the flat panel for a given field size $w$ to the dose measured by the ionization chamber for a given field size $w$ normalized to the corresponding ratio for a $10 \times 10 \mathrm{~cm}^{2}$ field. The air scatter factors range from 0.953 for a $5 \times 5 \mathrm{~cm}^{2}$ field to 1.067 for a $20 \times 20 \mathrm{~cm}^{2}$ field.

\subsection{Back Projection Formalism for EPID Dosimetry}

In Figure 3, the attenuator factor $A F(w, t)$ as a function of field side $w$ and phantom thickness $t$ is presented. The attenuator factor for intermediate attenuation thicknesses can be calculated by interpolating the corresponding measured data.

\subsection{Formalism for EBT2 Film Dosimetry}

The calibration curve of EBT2 films is presented in Figure 4. While, in Table 1 the conversion factors of surface dose to maximum dose $\left(C F_{\text {Surface Dose } \rightarrow D_{\max }}\right)$ are presented as a function of field side. Each factor $\left(C F_{\text {Surface Dose } \rightarrow D_{\max }}\right)$ is the mean value of the respective factors measured for an irradiated field size $w$ and a phantom of thickness $10 \mathrm{~cm}$ and $20 \mathrm{~cm}$.

\subsection{The Mid-Plane Dose by Phantom Verification Measurements}

The distribution of the EPID reconstructed doses ratios to the TPS calculated doses reveals a mean value of 0.993 and a standard deviation of 0.013 (Figure 5). While, the distribution of the ratios of the EBT2 film reconstructed doses to the TPS calculated doses reveals a mean value of 0.971 and a standard deviation of 0.010 (Figure 5).

The distribution of the ratios of the EPID reconstructed doses to the ionization chamber measured doses reveals a mean value of 0.983 and a standard deviation of 0.013 (Figure 6). While, the distribution of the ratios of 


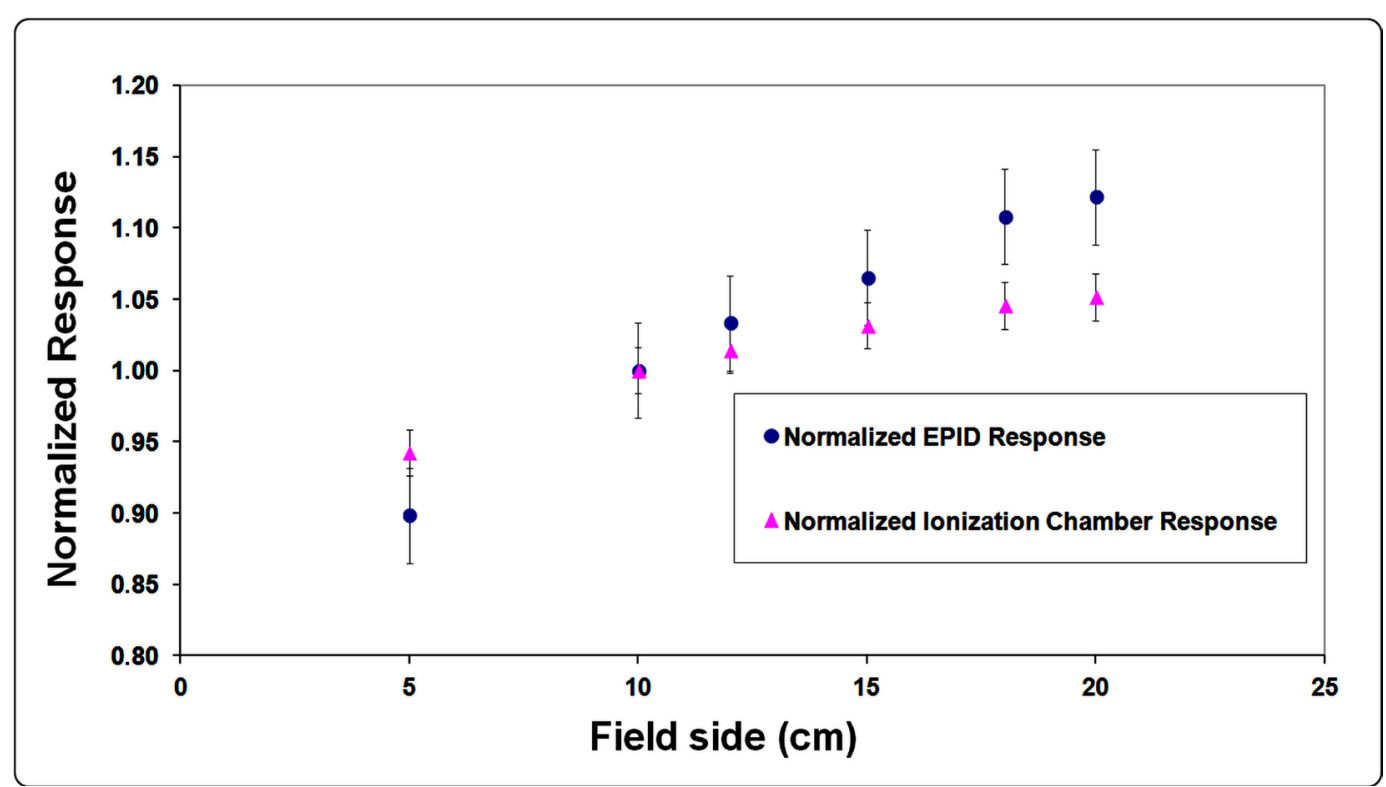

Figure 2. The normalized EPID and ionization chamber response as a function of field side.

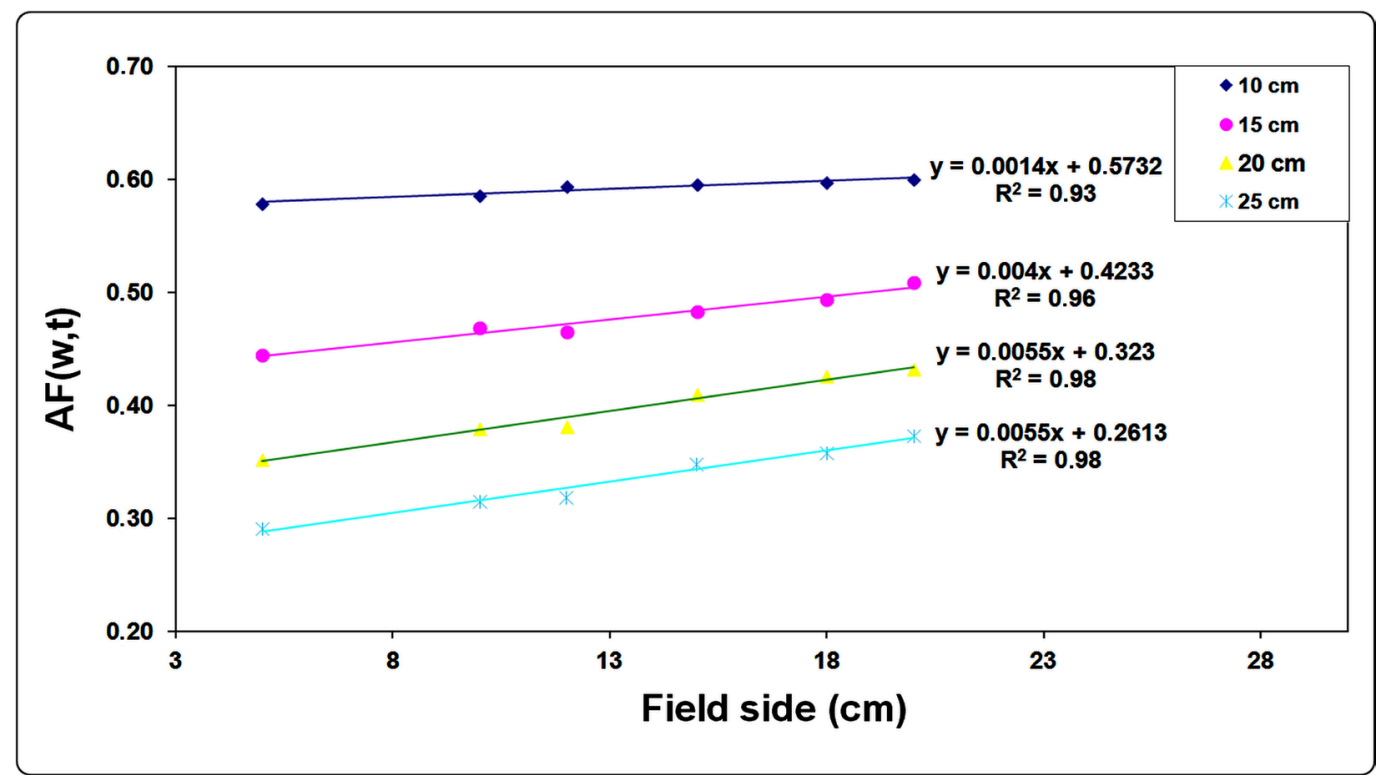

Figure 3. The attenuator factor $A(w, t)$ as a function of field side $w$ and phantom thickness $t$.

the EBT2 film reconstructed doses to the ionization chamber measured doses reveals a mean value of 0.962 and a standard deviation of 0.010 (Figure 6).

It is also raised that the discrepancy between the EPID method and the EBT2 film method is $2.2 \%$.

\subsection{In Vivo Measurements}

The ratio distribution of the EPID in vivo reconstructed doses to the TPS isocenter doses reveals a mean value of 1.008 and a standard deviation of 0.032 (Figure 7). While, the ratio distribution of the EBT2 film in vivo reconstructed doses to the TPS isocenter doses reveals a mean value of 0.998 and a standard deviation of 0.013 (Figure 7). These values are within the $\pm 5 \%$ which is the classical in vivo tolerance level [2], indicating the method's accuracy. It is also revealed that the discrepancy between EPID dosimetry and EBT2 film dosimetry is $1 \%$. 
Film Calibration

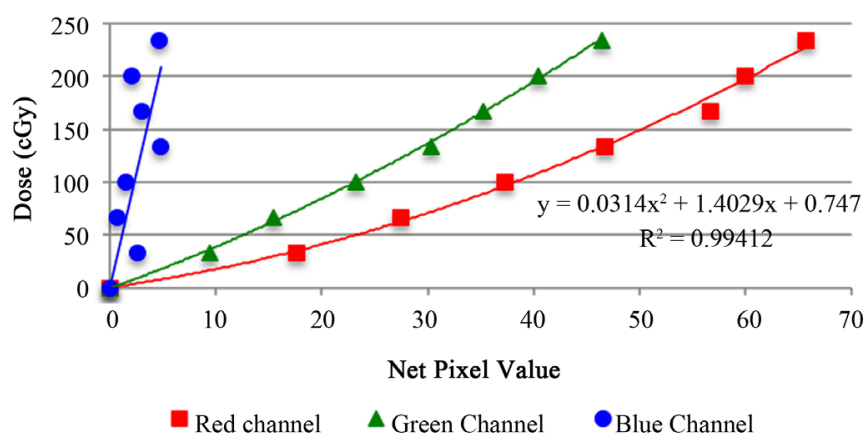

Figure 4. EBT2 film calibration curve. The red channel is considered appropriate for the image analysis. The fitting function refers to the red channel.

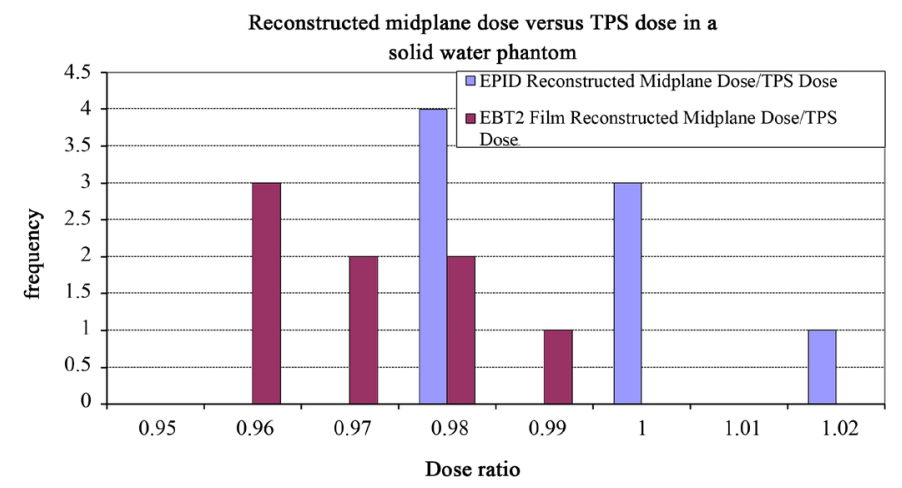

Figure 5. The distribution of the ratios of the EPID reconstructed mid-plane doses $D_{P}^{\mathrm{EPID}}(d)$ to the treatment planning calculated mid-plane doses $D_{P}^{\mathrm{TPS}}(d)$ and the distribution of the ratios of the EBT2 film reconstructed mid-plane doses $D_{P}^{\mathrm{EBT2}}(d)$ to the treatment planning calculated mid-plane doses $D_{P}^{\mathrm{TPS}}(d)$ in a homogenous solid water phantom.

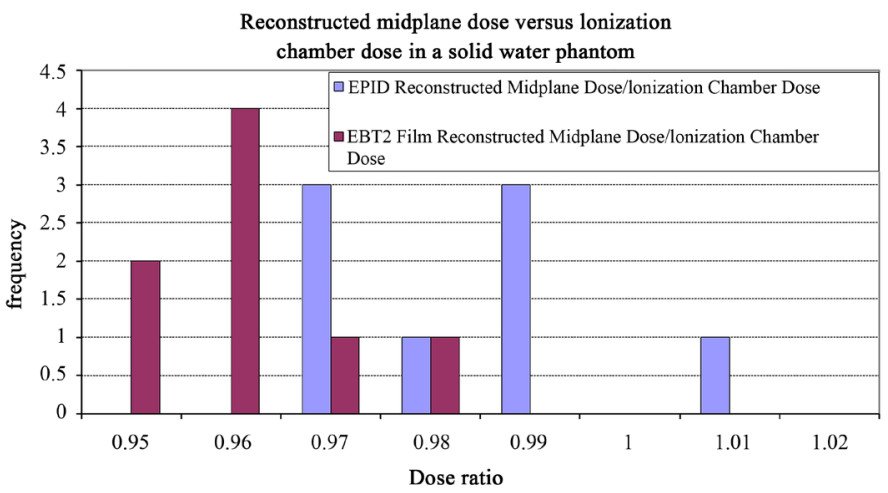

Figure 6. The distribution of the ratios of the EPID reconstructed mid-plane doses $D_{P}^{\mathrm{EPID}}(d)$ to the ionization chamber measured midplane doses and the distribution of the ratios of the EBT2 film reconstructed mid-plane doses $D_{P}^{\mathrm{EBT2}}(d)$ to the ionization chamber measured mid-plane doses in a homogenous solid water phantom. 


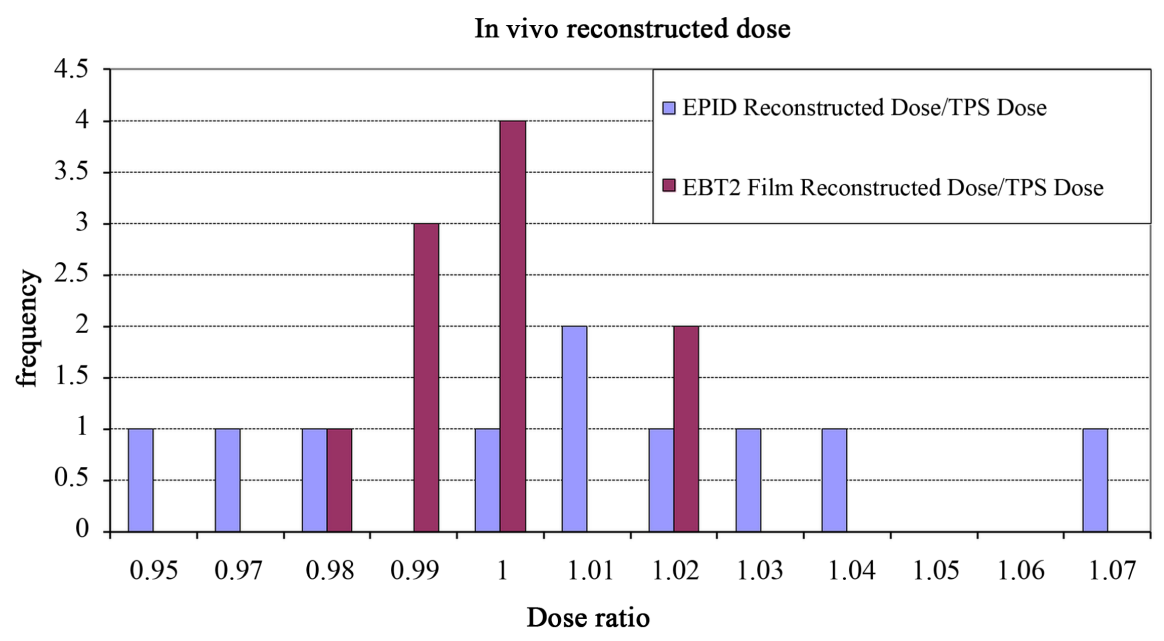

Figure 7. The distribution of the ratios of the EPID in vivo reconstructed doses to the treatment planning calculated isocenter doses and the distribution of the ratios of the EBT2 film in vivo reconstructed doses to the treatment planning calculated isocenter doses.

Table 1. The conversion factor of surface dose to maximum dose.

\begin{tabular}{cccc}
\hline Field side $(\mathrm{cm})$ & Conversion factor of surface dose to maximum dose $C F_{\text {Surfac Dose } \rightarrow D_{\max }}$ & $(\mathrm{SD})$ \\
\hline 5 & 8.42 & 0.94 \\
10 & 5.94 & 0.52 \\
15 & 4.88 & 0.37 \\
20 & 3.69 & 0.24 \\
\hline
\end{tabular}

\section{Discussion}

In this study an easily applicable EPID dosimetry method is proposed for clinical practice, demanding a small number of data measurements (buildup, ghosting effects, calibration, field size dependence, thickness dependence).

In order to define formalism for EPID dosimetry it is necessary to determine the flat panel's inherent dosimetric characteristics. Firstly, it was determined that the additional build-up layer is $0.4 \mathrm{~cm}$ solid water. This result is in accordance with the results of G. V. Menon and R. S. Sloboda [7]. They have studied an a-Si EPID of a different vendor and have found that the maximum EPID response occurs for a thickness of $0.5 \mathrm{~cm}$ solid water for a $6 \mathrm{MV}$ photon beam.

In this study ghosting effects were investigated. It was found that in low doses the normalized EPID response deviates from the ideal linear response due to ghosting effects. This behavior has also been commented by other authors who have studied the flat panel characteristics of the same [3] or other vendors' [4] [8] [9]. The ghosting correction factor was introduced to correct the EPID response for non-linear behavior. After correcting the measurements with the $G(t)$ the linearity with the MUs was within $\pm 2 \%$ for the whole range of MUs.

Investigating the field size dependence of EPID response it was found that the EPID response differs significantly from the ionization chamber response for various field sizes, up to $6.7 \%$. This result can be attributed to the increased scattered irradiation created inside the flat panel's electronics. To take into account this discrepancy, we introduced the EPID air scatter factor used in all further EPID measurements.

Finally, investigating the EPID response under attenuation conditions the attenuation factor was introduced. It was found that for the same attenuator thickness the attenuation is slightly increased with increasing field size because of the increasing scattered photons.

Once the inherent and dosimetric characteristics of the flat panel were determined, a dosimetric model, which calculates the dose in the absorber on the central axis of the photon beam, was developed. The dosimetric model is based on the back projection approach and takes into account: 
1) The low energy photons with the use of the additional buildup layer

2) The image lag and gain ghosting effects with the use of the ghosting correction factor, $G(t)$

3) The scattered irradiation created inside the flat panel's electronics with the use of the EPID air scatter factor, $S_{\mathrm{EPID}}^{\mathrm{AIR}}(w)$ and

4) The attenuation of photon beam due to the presence of various thicknesses absorber in the path of the beam with the use of the attenuation factor, $A F(w, t)$.

The performance of the proposed method was firstly assessed with phantom measurements. According to phantom verification measurements, the mean dispersion of the ratios distribution of the EPID reconstructed doses to the ionization chamber and TPS measured doses is $1.7 \%$ and $0.7 \%$ respectively, indicating that the proposed method is accurate and can be further applied on patients for in vivo measurements. The results also revealed a $1 \%$ systematic difference between the doses measured with the ionization chamber and the corresponding doses calculated by the TPS, which can be attributed to the linac output fluctuations $( \pm 1 \%)$.

Once the method was validated, it was applied on ten prostate patients treated with $3 \mathrm{D}$ conformal plans. The EPID reconstructed doses were compared to the corresponding TPS calculated doses. The mean dispersion of the ratio of the EPID in vivo reconstructed doses to the TPS doses is $0.8 \%$. According to the results shown in Figure 7 the agreement between planned and EPID measured doses is within $\pm 4 \%$ including the uncertainty of the method (1.7\%), the determination of patient's thickness, the determination of the equivalent square field and the uncertainties caused by the patient's physiological movements. It is underlined that in only one case, the EPID verification method was out of the acceptable tolerance level (7\%). This discrepancy caused by the presence of gas cavities in patient's abdomen which was not recorded in the initial CT scan.

Comparing the EPID and the EBT2 dosimetry methods the phantom measurements revealed a difference of $+2.2 \%$ which can be attributed to the uncertainty in determination of the surface dose to the maximum dose conversion factors. On the other hand, the deviation between the EPID dosimetry method and the EBT2 film dosimetry method is $1 \%$ for the in vivo measurements. This difference is due to 1 ) the uncertainty in determination of the surface dose to the maximum dose conversion factors and 2) the difficulty in EBT2 film positioning on the patient's skin. Regarding the case which was out of the acceptable tolerance level, the presence of the gas cavities does not affect the EBT2 film measurement because the entrance dose measurements are independent from patient's anatomy. This fact indicates an advantage of the EPID dosimetry method over the EBT2 film method. Moreover, the EPID dosimetry method can be applied for all the irradiation fields without interrupting the treatment and without the patients coming into contact with the flat panel. Finally, the EPID images are available immediately after patient irradiation.

Many authors have proposed other EPID dosimetry methods. Some of them (Chytyk-Praznik et al., Royer et al., Gimeno et al.) have proposed methods which are based on Monte Carlo calculations or sophisticated software [18]-[20]. However, the aim of this study was to propose an easily applicable EPID dosimetry method without the use of any sophisticated software. Two main research groups (Piermattei et al., Francois et al.) have also proposed other easily applicable methods [21] [22]. Particularly, with regards to the Piermattei et al. approach, which is based on the use of correlation functions, the average ratio of the reconstructed dose at the isocenter to the TPS dose for pelvic tumors was within 1\%, in agreement with the mean values reported in this study [21]. Regarding the Francois et al. approach, who also proposed an easily applicable in vivo verification method on the central axis using the back-projection approach, the mean dose ratio for 20 3DCRT prostate treatments was also within 1\% [22]. However, our method takes into account 1) the electronic equilibrium and the low energy photon attenuation with the addition of the appropriate build-up layer on the EPID surface and 2) the ghosting effects with the implementation of the ghosting correction factor $\mathrm{G}(\mathrm{t})$. On the other hand, the proposed method does not use the transmission functions $\left(\mathrm{f}(\mathrm{d}, \mathrm{l}), \mathrm{TMR}^{\mathrm{t}}\right)$, which have been used by Piermattei et al. and Francois et al. respectively, thus reducing the set of measurements considerably. The use of these functions was not adopted because a) the use of the build-up layer assures the elimination of the scattered radiation reaching the detector and $b$ ) the scattered radiation is negligible for the air gaps met in clinical practice $(>20 \mathrm{~cm})[8]$ [13].

\section{Conclusions}

An easily applicable EPID dosimetry method based on the back-projection approach was proposed. The method is accurate, easily applicable to all types of flat panels given that inherent characteristics of each specific flat 
panel have been determined and the calculation of the dose can be performed without any sophisticated software.

The major advantages of the EPID dosimetry method over EBT2 film dosimetry are: 1) the EPID takes into account the patient's anatomy and 2) the method is not time consuming. While further investigation may take advantage of the 2D dosimetry information recorded on the flat panel.

The presented EPID dosimetry method is intended to be used in more complex techniques. The perspective of the method application on the off axis points is also under investigation.

\section{References}

[1] Wendling, M., Louwe, R.J.W., McDermott, L.N., Sonke, J.J., Van Herk, M. and Mijnheer, B.J. (2006) Accurate Two-Dimensional IMRT Verification Using a Back-Projection EPID Dosimetry Method. Medical Physics, 33, 259273. http://dx.doi.org/10.1118/1.2147744

[2] International Atomic Energy Agency (2013) Development of Procedures for in Vivo Dosimetry in Radiotherapy. IAEA Human Health Report No. 8.

[3] Nijsten, S.M.J.J.G., Van Elmpt, W.J.C., Jacobs, M., Mijnheer, B.J., Dekker, A.L.A.J., Lambin, P., et al. (2007) A Global Calibration Model for a-Si EPIDs Used for Transit Dosimetry. Medical Physics, 34, 3872-3884. http://dx.doi.org/10.1118/1.2776244

[4] McDermott, L.N., Louwe, R.J.W., Sonke, J.J., Van Herk, M.B. and Mijnheer, B.J. (2004) Dose-Response and Ghosting Effects of an Amorphous Silicon Electronic Portal Imaging Device. Medical Physics, 31, 285-295. http://dx.doi.org/10.1118/1.1637969

[5] McDermott, L.N., Wendling, M., Sonke, J.J., Van Herk, M. and Munheer, B.J. (2007) Replacing Pretreatment Verification with in Vivo EPID Dosimetry for Prostate IMRT. International Journal of Radiation Oncology*Biology*Physics, 67, 1568-1577. http://dx.doi.org/10.1016/j.ijrobp.2006.11.047

[6] Herman, M.G., Balter, J.M., Jaffray, D.A., McGee, K.P., Munro, P., Shalev, S., et al. (2001) Clinical Use of Electronic Portal Imaging: Report of AAPM Radiation Therapy Committee Task Group 58. Medical Physics, 28, 712-737. http://dx.doi.org/10.1118/1.1368128

[7] Menon, G.V. and Sloboda, R.S. (2003) Compensator Quality Control with an Amorphous Silicon EPID. Medical Physics, 30, 1816-1824. http://dx.doi.org/10.1118/1.1584040

[8] Chang, J., Mageras, G.S., Ling, C.C. and Lutz, W. (2001) An Iterative EPID Calibration Procedure for Dosimetric Verification That Considers the EPID Scattering Factor. Medical Physics, 28, 2247-2257. http://dx.doi.org/10.1118/1.1410122

[9] McDermott, L.N., Nijsten, S.M.J.J.G., Sonke, J.J., Partridge, M., Van Herk, M. and Mijnheer, B.J. (2006) Comparison of Ghosting Effects for Three Commercial a-Si EPIDs. Medical Physics, 33, 2448-2451. http://dx.doi.org/10.1118/1.2207318

[10] Fidanzio, A., Cilla, S., Greco, F., Gargiulo, L., Azario, L., Sabatino, D., et al. (2011) Generalized EPID Calibration for in Vivo Transit Dosimetry. Physica Medica, 27, 30-38. http://dx.doi.org/10.1016/j.ejmp.2010.02.002

[11] Van Esch, A., Depuydt, T. and Huyskens, D.P. (2004) The Use of an aSi-Based EPID for Routine Absolute Dosimetric Pre-Treatment Verification of Dynamic IMRT Fields. Radiotherapy and Oncology, 71, 223-234. http://dx.doi.org/10.1016/j.radonc.2004.02.018

[12] El-Mohri, Y., Antonuk, L.E., Yorkston, J., Jee, K.W., Maolinbay, M., Lam, K.L., et al. (1999) Relative Dosimetry Using Active Matrix Flat-Panel Imager (AMFPI) Technology. Medical Physics, 26, 1530-1540. http://dx.doi.org/10.1118/1.598649

[13] Zhu, Y., Jiang, X.Q. and Van Dyk, J. (1995) Portal Dosimetry Using a Liquid Ion Chamber Matrix: Dose Response Studies. Medical Physics, 22, 1101-1106. http://dx.doi.org/10.1118/1.597502

[14] Mohammadi, M. and Bezak, E. (2006) Two-Dimensional Transmitted Dose Measurements Using a Scanning Liquid Ionization Chamber EPID. Physics in Medicine and Biology, 51, 2971-2985. http://dx.doi.org/10.1088/0031-9155/51/11/019

[15] Alva, H., Mercado-Uribe, H., Rodriguez-Villafuerte, M. and Brandan, M.E. (2002) The Use of a Reflective Scanner to Study Radiochromic Film Response. Physics in Medicine and Biology, 47, 2925-2933. http://dx.doi.org/10.1088/0031-9155/47/16/308

[16] Hartmann, B., Martisikova, M. and Jakel, O. (2010) Homogeneity of Gafchromic EBT2 Film. Medical Physics, 37, 1753-1756. http://dx.doi.org/10.1118/1.3368601

[17] Alves, V., Cardoso, S. and da Silva, A. (2013) Gafchromic EBT2 Dosimetry via Robust Optimization. Computer Physics Communications, 184, 1708-1716. http://dx.doi.org/10.1016/j.cpc.2013.02.023 
[18] Chytyk-Praznik, K., VanUytven, E., vanBeek, T.A., Greer, P.B. and McCurdy, B.M. (2013) Model-Based Prediction of Portal Dose Images during Patient Treatment. Medical Physics, 40, Article ID: 031713. http://dx.doi.org/10.1118/1.4792203

[19] Royer, P., Marchesi, V., Rousseau, V., Buchheit, I., Wolf, D., Peiffert, D. and Noel, A. (2014) Evaluation of Transit in Vivo Dosimetry Using Portal Imaging and Comparison with Measurements Using Diodes. Cancer/Radiotherapie, 18, 183-190. http://dx.doi.org/10.1016/j.canrad.2014.03.006

[20] Gimeno, J., Pujades, M.C., Garcia, T., Carmona, V., Lliso, F., Palomo, R., et al. (2014) Commissioning and Initial Experience with a Commercial Software for in Vivo Volumetric Dosimetry. Physica Medica, 30, 954-959. http://dx.doi.org/10.1016/j.ejmp.2014.06.004

[21] Piermattei, A., Fidanzio, A., Azario, L., Grimaldi, L., D’Onofrio, G., Cilla, S., et al. (2007) Application of a Practical Method for the Isocenter Point in Vivo Dosimetry by a Transit Signal. Physics in Medicine and Biology, 52, 5101-5117. http://dx.doi.org/10.1088/0031-9155/52/16/026

[22] Francois, P., Boissard, P., Berger, L. and Mazal, A. (2011) In Vivo Dose Verification from Back Projection of Transit Dose Measurement on the Central Axis of Photon Beams. Physica Medica, 27, 1-10.

http://dx.doi.org/10.1016/j.ejmp.2010.06.002 